\title{
In vivo ultrasound assessment of respiratory function of abdominal muscles in normal subjects
}

\author{
G. Misuri*, S. Colagrande**, M. Gorini+, I. Iandelli+', \\ M. Mancini++, R. Duranti ${ }^{++}$, G. Scano ${ }^{++}$
}

In vivo ultrasound assessment of respiratory function of abdominal muscles in normal subjects. G. Misuri, S. Colagrande, M. Gorini, I. Iandelli, M. Mancini, R. Duranti, G. Scano. CERS Journals Ltd 1997.

ABSTRACT: Ultrasonography has recently been proposed for assessing changes in thickness and motion of the diaphragm during contraction in humans. Data on ultrasound assessment of abdominal muscles in humans are scarce. We therefore investigated the changes in thickness and the relevant mechanical effects of abdominal muscles using this technique during respiratory manoeuvres in normal subjects.

We evaluated the thickness of the abdominal muscle layers in six normal male subjects (aged 26-36 yrs) using a 7.5 MHz B-mode ultrasound transducer. Gastric $(P \mathrm{~g})$ and mouth pressures, muscle thickness of external oblique $(\mathrm{EO})$, internal oblique (IO), transversus abdominis (TA) and rectus abdominis (RA) were assessed at functional residual capacity (FRC), residual volume (RV), total lung capacity (TLC), during progressive (PEEs) and maximal expiratory efforts (MEEs) against a closed airway and during homolateral (HTR) and contralateral (CTR) trunk rotation.

Abdominal muscle thickness was found to be reproducible (coefficient of variation and two-way analysis of variance). Compared to FRC, the thickness of IO, TA and RA significantly increased at RV and during MEEs, whereas EO remained unchanged; at TLC, the thickness of IO and TA significantly decreased. During PEEs, a significant relationship between increase in $P g$ and $\mathrm{TA}$ thickness was observed in all subjects, the thickness of the other abdominal muscles being inconsistently related to $\mathrm{Pg}$. Finally, a significant increase in the thickness of IO and EO was found during HTR and CTR, respectively.

We conclude that during maximal expiratory manoeuvres, transversus abdominis, internal oblique and rectus abdominis thickened similarly. Transversus abdominis seems to be the major contributor in generating abdominal expiratory pressure during progressive expiratory efforts. External oblique seems to be preferentially involved during trunk rotation. These results suggest the possible value of studying the abdominal muscles by ultrasonography in various respiratory disorders.

Eur Respir J 1997; 10: 2861-2867.

In recent years, it has been shown both in the dog [1-4] and humans [5-9] that abdominal expiratory muscles do not operate as a unit. In particular, electromyographic recordings from individual abdominal muscles using needle $[5,8]$ or fine wire electrodes $[6,7,9]$ have shown that during breathing in humans, transversus abdominis (TA) and internal oblique (IO) are recruited in preference to the superficial muscle layers. In all these studies, abdominal muscle activation was detected by intramuscular electrodes, an appropriate but invasive technique not suitable in a clinical setting.

Recent studies have investigated the inspiratory muscles in humans by means of ultrasound. This method has been shown reliably to measure changes in diaphragm thickness during contraction $[10,11]$ and has been used to assess the relationship between lung volume and diaphragmatic thickness [10, 12-14]. In contrast, to our knowledge, ultrasonographic data on abdominal muscles in humans are scarce both for normal subjects $[5,6]$ and for a number of respiratory diseases in which
*Fondazione Pro Juventute Don C. Gnocchi, Pozzolatico, **Dept of Radiology University of Florence, +Pulmonary Intensive Care Unit, Policlinico di Careggi, and ${ }^{++}$Section of Pneumology, Istituto di Medicina Interna ed Immunoallergologia, University of Florence, Italy.

\section{Correspondence: G. Scano}

Istituto di Medicina Interna ed Immunoallergologia

Università degli Studi di Firenze

V.le G.B. Morgagni

85 - 50134 Firenze

Italy

Keywords: Abdominal muscles muscle thickness

ultrasound

Received: October 211996 Accepted after revision July 11997 abdominal muscles contribute importantly to respiration [8].

Therefore, in a preliminary study we have employed ultrasonography in normal humans to assess the thickness and the relevant mechanical effects of the abdominal muscles during several respiratory manoeuvres.

\section{Methods}

\section{Subjects}

We studied six healthy male subjects, aged 26-36 yrs. None had ever smoked nor had any history of cardiopulmonary or neuromuscular diseases, and their spirograms were within normal limits. All were familiar with respiratory manoeuvres. The protocol of the study was approved by the University Ethics Committee and written informed consent was obtained from each subject. 
a)
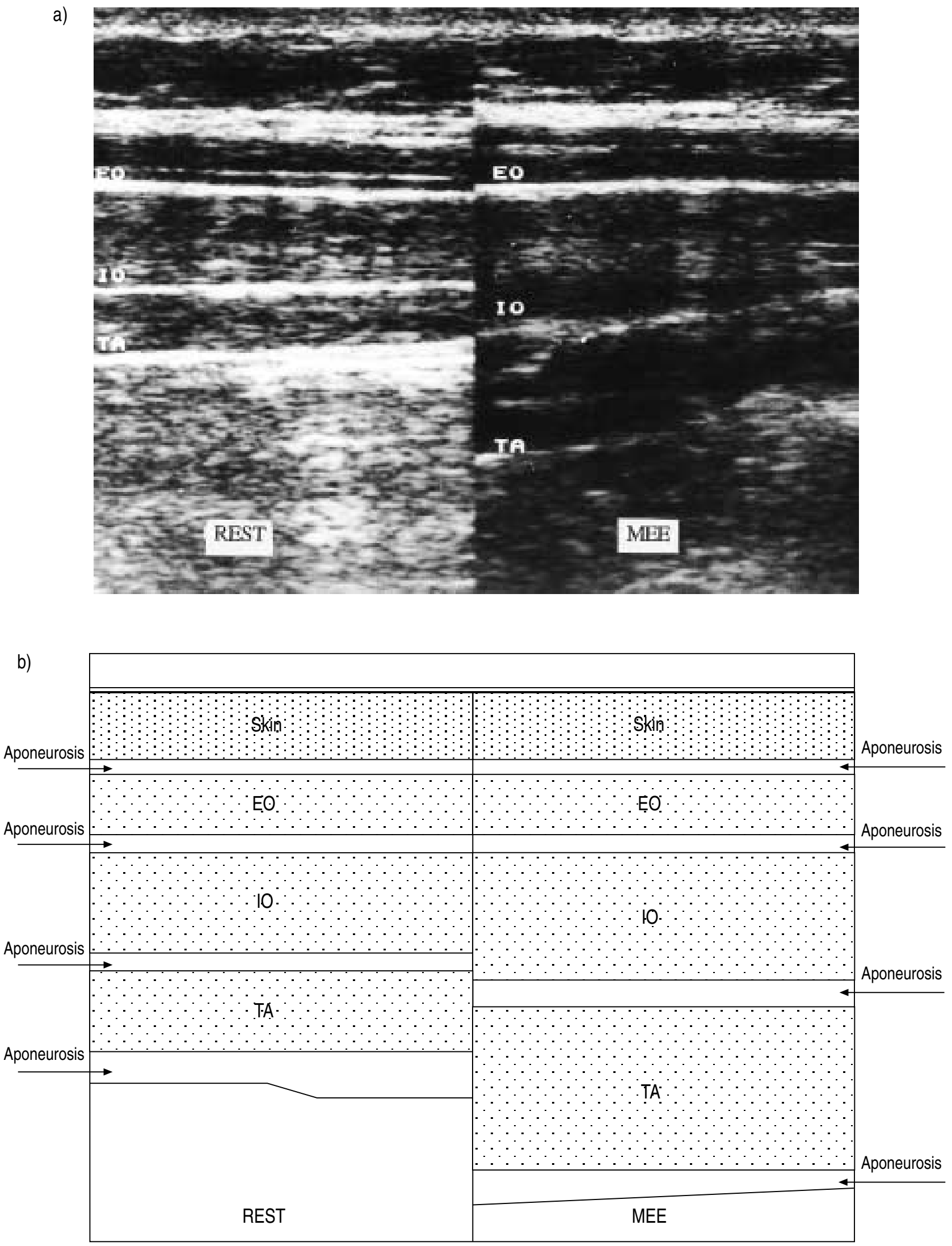

Fig. 1. - a) Ultrasound image of the three muscles of the lateral wall of the abdomen at functional residual capacity (FRC) (left side) and during a maximal expiratory effort (MEE) manoeuvre (right side) in a representative subject. Note that TA and IO thickened markedly during the manoeuvre, whereas the thickness of EO remained substantially unchanged. b) A schematic representation of ultrasound image illustrating the layers composing the lateral abdominal wall. EO: external oblique; IO: internal oblique; TA: transversus abdominis. 


\section{Measurements}

Subjects were seated comfortably in a high-backed armchair at an angle of $90^{\circ}$. They breathed through a mouthpiece via a Fleisch No. 3 pneumotachograph (Beckman Instruments Inc., Schiller Park, IL, USA) attached to a rigid and fixed bracket, so that no change in position was permitted, and they were instructed to avoid movement as much as possible. The flow signal was integrated to give volume.

One pair of linearized magnetometers attached in the midline $2 \mathrm{~cm}$ above the umbilicus were used to assess change in anteroposterior (AP) diameter of the abdomen. Changes in AP diameter during tidal breathing were obtained in arbitrary units. To normalize data, abdominal excursion during forced expiratory manoeuvres was expressed as a percentage of the abdominal excursion during tidal breathing.

Oesophageal $(P \mathrm{pl})$ and gastric $(P \mathrm{~g})$ pressures were measured using a conventional balloon-catheter system positioned in the midoesophagus and in the stomach, respectively, as described previously [15]. Each ballooncatheter system was connected to a differential pressure transducer (Validyne, Northridge, CA, USA). During quiet breathing, oesophageal and gastric balloons were inflated with 0.5 and $1 \mathrm{~mL}$ of air, respectively, whereas during forced expiratory efforts the balloons were inflated with $2.5 \mathrm{~mL}$ of air, in order to avoid their collapse. A third differential pressure transducer, connected to the mouthpiece, was used to measure mouth pressure.

During progressive expiratory efforts, mouth pressure was displayed on an oscilloscope (Tektronix 2225; Tektronix Corp, Beaverton, OR, USA), to provide a visual feedback of pressure generated and allow subjects to develop a predetermined value of expiratory mouth pressure. Respiratory manoeuvres (residual volume (RV) and total lung capacity (TLC)) were also displayed on the oscilloscope to provide a feedback of pulmonary volume.

Volume, flow, magnetometer and pressure signals were recorded on a multichannel chart recorder (TA 4000; Gould, Valley View, OH, USA).

Abdominal muscle thickness was assessed using a 7.5 MHz transducer (SSD-650; Aloka Co. Ltd., Tokyo, Japan) in B-mode condition. Before starting the study we carried out a preliminary session to define where to position the ultrasound probe. We looked for a position that would be easily identifiable by anatomical landmarks and allow continuous ultrasound visualization of each muscle boundary during thickening. Based on previous studies [5, 6], for the lateral wall of the abdomen we placed the probe midway between the costal margin and the iliac crest, along the right anterior axillary line. For rectus abdominis (RA), the probe was placed $2-3 \mathrm{~cm}$ above the umbilicus, $2-3 \mathrm{~cm}$ from the midline. Great care was taken to keep the probe perpendicular to the abdominal surface throughout the manoeuvres. During quiet breathing at the end of expiration (at functional residual capacity (FRC)), the ultrasound image was frozen on the left side of the video; the distance between the inner and outer surface of each muscle was then measured. Each muscle was identified easily thanks to separating clear bright lines representing muscle sheaths. Subsequently, the images of the abdominal mus- cles were displayed continuously on the right side of the video until the end of each manoeuvre, when the chart recorder was stopped and simultaneously the ultrasound image was frozen (fig. 1).

\section{Protocol}

The thickness of the three muscles of the lateral wall of the abdomen (external oblique (EO), IO and TA) and of RA was evaluated during the following situations: 1) under control conditions at FRC; 2) during breath holding with open airway both at RV and at TLC; 3) during maximal expiratory efforts (MEEs) at FRC with open glottis against a closed airway; 4) during progressively increasing expiratory efforts (PEEs) at FRC with open glottis against a closed airway.

Subjects performed RV, TLC, MEE and PEE manoeuvres in two different sessions, the first for EO, IO, TA and the second for RA muscle thickness assessment, respectively. During each session, from three to five RV and TLC manoeuvres were performed, and the mean value of thickness was used for analysis. The MEE manoeuvre was repeated until three values with a variability $<5 \%$ were obtained. The results presented are those of the abdominal muscle thickness in the ultrasound image obtained during the effort with the highest MEE value. From 8-13 PEEs were carried out in random order. During the second PEE session, subjects were asked to reproduce values of mouth pressure similar to the first session. The thickness of EO, IO and TA was also evaluated at FRC during homolateral (HTR) and contralateral (CTR) trunk rotation against a resistance; between three and five manoeuvres were carried out.

\section{Data analysis}

The repeatability of measurements of abdominal muscle thickness was studied at FRC, RV and TLC by calculating coefficients of variation and by two-way analysis of variance (ANOVA). The thickness of the four abdominal muscles at FRC was compared using one-way ANOVA, whereas the thickness of each abdominal muscle before (at FRC) and at the end of each manoeuvre was compared by Student's paired t-test. The relationship between $\mathrm{Pg}$ developed during PEE and the corresponding thickness of abdominal muscles was tested by regression analysis. A p-value less than 0.05 was taken to be significant. Values are mean \pm SD.

\section{Results}

Thickness measurements of each abdominal muscle at FRC, RV and TLC were repeatable with coefficients of variation ranging $0-15.7 \%$ (table 1). Moreover, for each muscle, between-subject variability was highly significant ( $F$ ranging 8.1-273, p-values ranging 0.003$0.00001)$ whereas intrasubject variability was not ( $F$ ranging $0.54-3.2$, $\mathrm{p}$-values ranging $0.66-0.06$ ).

At FRC, the thickness of EO, IO, TA and RA was $5.5 \pm 1.7,11.1 \pm 3.8,5.8 \pm 1.3,11 \pm 4.3 \mathrm{~mm}$, respectively (fig. 2). Internal oblique and RA were thicker than EO and TA $(\mathrm{p}<0.004$, one-way ANOVA). 
Table 1. - Coefficients of variation of thickness of each abdominal muscle at FRC, RV and TLC for each subject

\begin{tabular}{lccccc}
\hline \multirow{2}{*}{ Subject } & Condition & \multicolumn{4}{c}{ Abdominal muscles } \\
\cline { 3 - 6 } No. & & EO & IO & TA & RA \\
\hline 1 & FRC & 0 & 0 & 0 & 0 \\
& RV & 0 & 2.0 & 4.3 & 7.1 \\
& TLC & 0 & 15.7 & 0 & 0 \\
2 & FRC & 9.2 & 12.3 & 10.3 & 3.4 \\
& RV & 11.1 & 1.9 & 0 & 1.8 \\
& TLC & 0 & 0 & 0 & 0 \\
3 & FRC & 0 & 0 & 0 & 0 \\
& RV & 0 & 0 & 13 & 4.3 \\
& TLC & 0 & 0 & 0 & 0 \\
4 & FRC & 6.0 & 11.2 & 15.6 & 10.8 \\
& RV & 7.7 & 3.0 & 4.3 & 3.7 \\
5 & TLC & 14.3 & 0 & 14.3 & 0 \\
5 & FRC & 0 & 0 & 0 & 0 \\
& RV & 0 & 0 & 0 & 0 \\
6 & TLC & 9.1 & 5.9 & 9.1 & 9.1 \\
6 & FRC & 0 & 0 & 0 & 0 \\
& RV & 9.8 & 6.3 & 15.2 & 5.7 \\
& TLC & 10.5 & 6.8 & 10.5 & 4.7 \\
\hline
\end{tabular}

FRC: functional residual capacity; RV: residual volume; TLC: total lung capacity; EO: external oblique; IO: internal oblique; TA: transversus abdominis; RA: rectus abdominis.
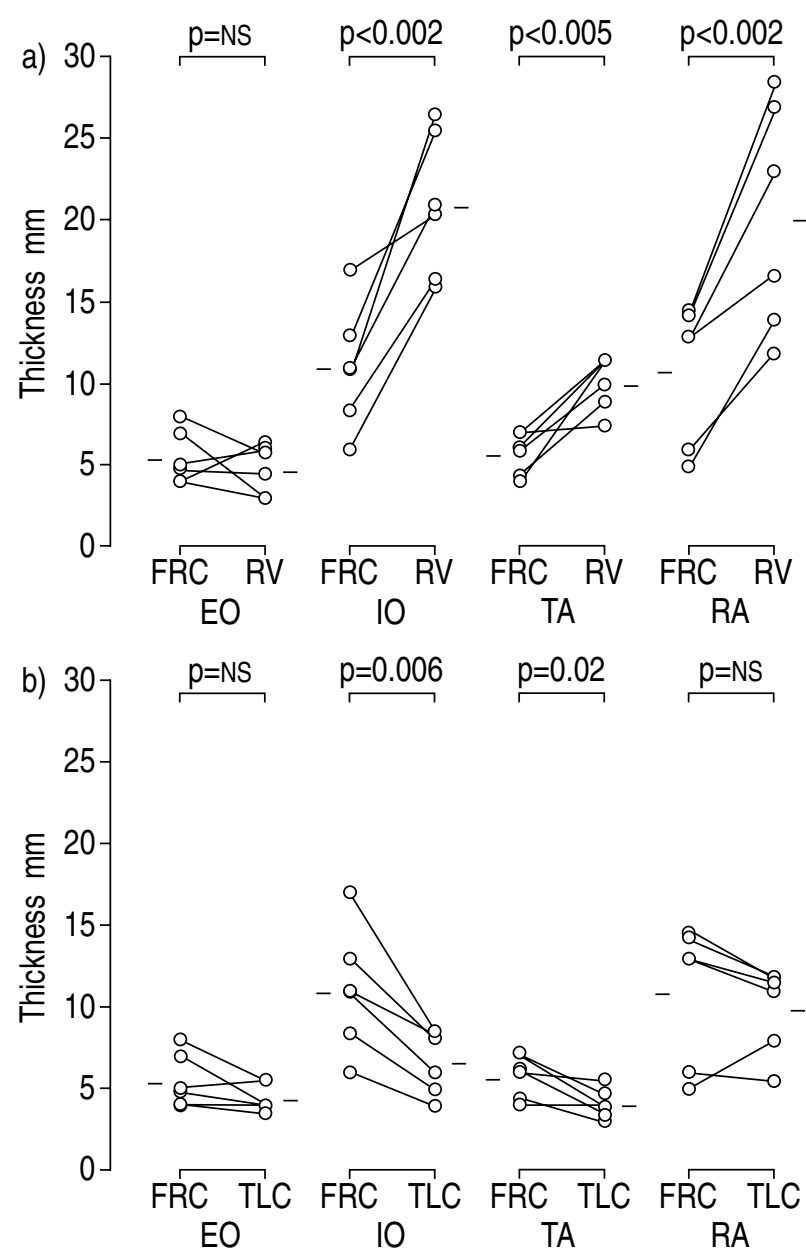

Fig. 2. - Changes in thickness of EO, IO, TA, and RA: a) from FRC to RV; and b) from FRC to TLC in six normal subjects. Open circles indicate individual values. Bars indicate means. Ns: nonsignificant. For further definitions see legend to table 1 .

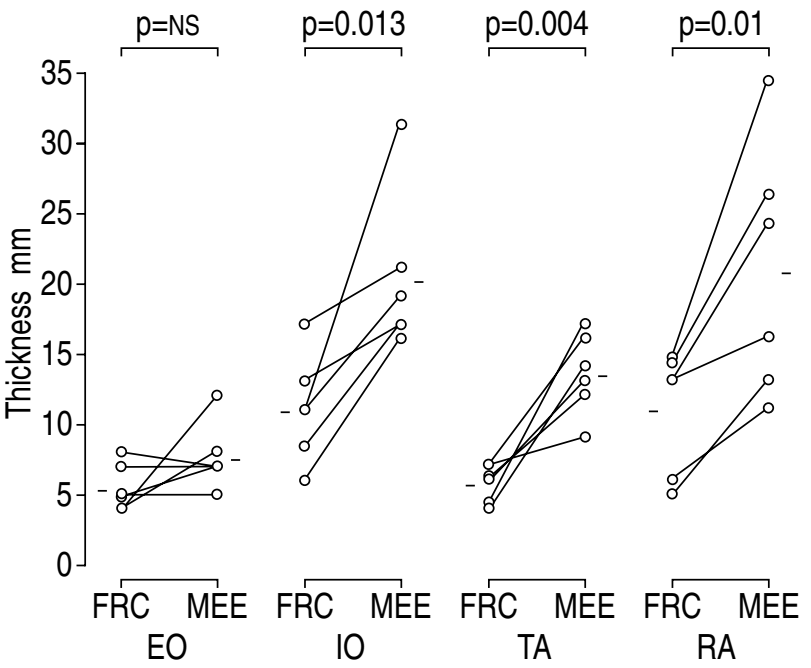

Fig. 3. - Thickness of EO, IO, TA, and RA during resting conditions (FRC) and a maximal expiratory effort (MEE) manoeuvre. Symbols as in figure 2. Ns: nonsignificant. For further definitions see legend to table 1.
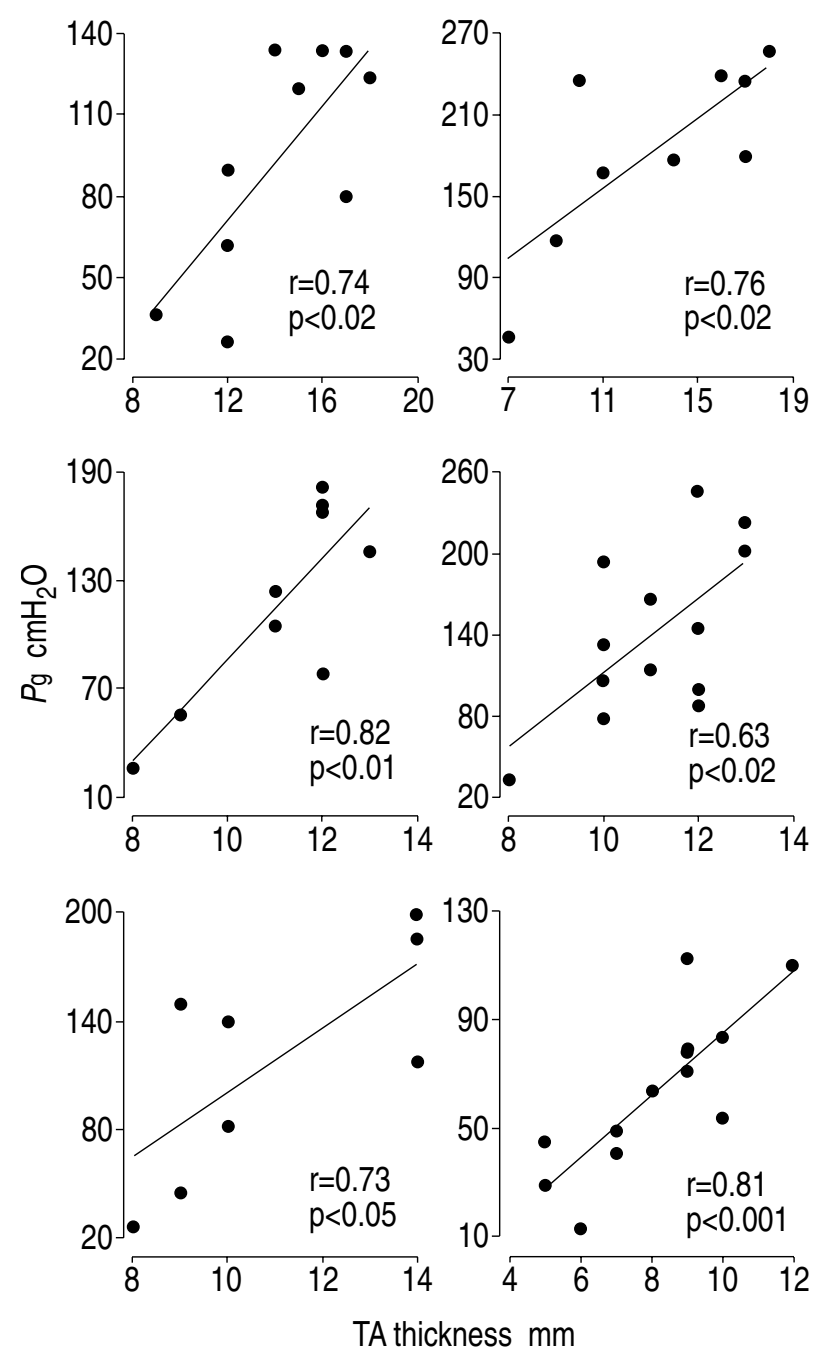

Fig. 4. - Plots of gastric pressure $(P \mathrm{~g})$ versus thickness of transversus abdominis (TA) in the six subjects during progressive expiratory efforts. 

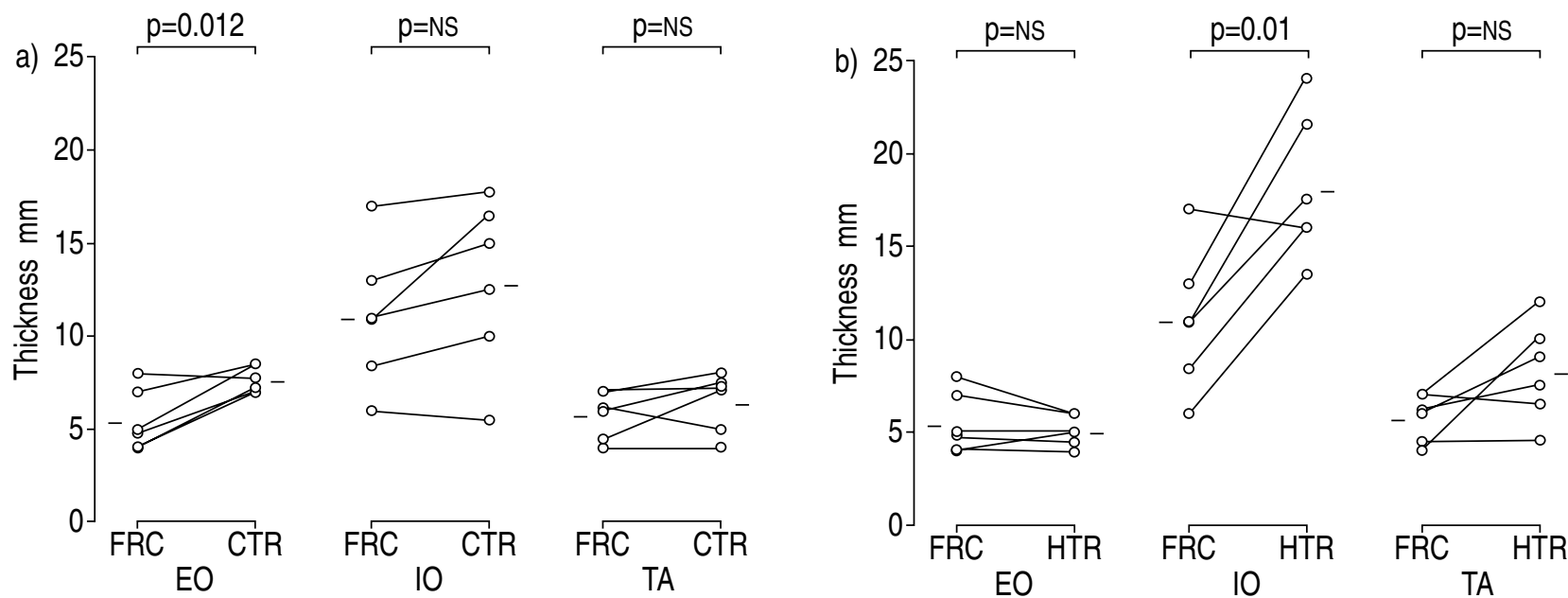

Fig. 5. - Changes in thickness of EO, IO, and TA during: a) contralateral trunk rotation (CTR); and b) homolateral trunk rotation (HTR). Symbols as in figure 2. Ns: nonsignificant. For further definitions see legend to table 1.

At RV compared to FRC a significant increase in thickness was found for IO, TA and RA, the changes in EO thickness being nonsignificant (fig. 2a).

From FRC to TLC the thickness of IO and TA decreased significantly, whereas the thickness of EO and RA remained substantially unchanged (fig. $2 b$ ).

During the MEE manoeuvre, mouth pressure ranged $102-180 \mathrm{cmH}_{2} \mathrm{O}$. During maximum efforts, IO, TA and RA thickened significantly, whereas the thickness of EO did not change (fig. 3). In each subject, abdominal AP diameter decreased below FRC: $-129.2 \% \pm 53.7$ (range -58 to $-272 \%$ ) of changes during quiet breathing.

During PEE, a significant relationship was found between $P \mathrm{~g}$ and TA thickness in all subjects (fig. 4). In contrast, $P \mathrm{~g}$ was not related consistently to the thickness of the other abdominal muscles. $P \mathrm{~g}$ was related significantly to the thickness of EO in subjects No. 2, 3 , and 4; to IO in subjects No. 3, 5, and 6; and to RA in subjects No. 3, 4, and 6. As during MEE manoeuvres, in all subjects abdominal AP diameter decreased below FRC at the highest pressure values developed during PEE manoeuvres.

Finally, the thickness of EO and IO increased significantly during contralateral (fig. 5a) and homolateral (fig. 5b) trunk rotation, respectively.

\section{Discussion}

The main findings of this study can be summarized as follows: 1) ultrasound measurement of abdominal muscle thickness is highly repeatable; 2) during maximal expiratory manoeuvres (RV, MEE), TA, IO and RA are recruited preferentially to EO; 3) during PEEs, TA plays a major role in abdominal pressure generation; and 4) EO thickens consistently during CTR.

\section{Background and validity of the method}

Recently, a number of authors have assessed the respiratory muscles in humans, especially the diaphragm, using the ultrasound technique [10-13]. Both in M-mode and $\mathrm{B}$-mode, ultrasound provides an alternative to other more sophisticated techniques, such as computed tomography, for assessing thickness, configuration and displacement of the diaphragm [16]. Several investigators have found significant linear relationships between changes in lung volume and either diaphragm movement or change in thickness, studying either the zone of apposition $[12,13]$ or the posterior part of the diaphragm [14]. Despite growing interest in ultrasound assessment of the diaphragm, studies specifically devoted to the abdominal muscles in humans are scarce. In an electromyographic study on abdominal muscle activity in humans, STROHL et al. [6] reported some ultrasound data on changes in thickness of the muscles of the upper and lower anterolateral wall of the abdomen. More recently, in another electromyographic study, De TROYER et al. [5] used ultrasound in order to insert needle electrodes accurately in the abdominal muscles in humans and at the same time measured muscle thickness at rest.

The abdominal muscles should be easier to visualize than the diaphragm with ultrasound, since the skin is the only anatomical structure between the probe and the muscle layers. In addition, unlike the diaphragm [12], no technical errors due to transducer misalignment are expected, since the transducer face was always carefully held parallel to the abdominal skin surface and consequently to abdominal muscles throughout the manoeuvres we performed.

Before commenting on the results the implication of muscle thickening requires consideration. It is well known [17] that individual muscles operate as systems with nearly constant volume, and changes in muscle diameter, associated inversely with change in length, can be taken as an index of motor response. In other words, when a muscle is activated, it shortens and thickens; so, unless muscle is passively shortened, the increase in muscle thickness is an index of muscle activation and it has been used in our paper in this sense.

\section{Findings of this study}

The ultrasound measurement of abdominal muscle thickness was found to be highly reproducible as demonstrated by the low coefficients of variation. Once the position 
of the probe was located, for either the lateral abdominal muscles or the RA, we were able to check changes in muscle thickness throughout the protocol. This finding is in line with previous ultrasound studies devoted to the diaphragm $[10,12,14]$, and confirms the reliability of this technique. The mean values of lateral wall abdominal muscle thickness we found at rest are similar to those measured by others [5, 6]. DE TROYER et al. [5] reported mean values of 7.2, 11.5 and $4.6 \mathrm{~mm}$ for EO, IO and TA, respectively. The slight differences between our results and those of DE TROYER et al. [5] might be due to small differences either in the level of fitness or body size of the subjects or both. STROHL et al. [6] reported that the sum of relaxed thickness of the three muscles of the upper abdominal wall ranged 11-20 $\mathrm{mm}$, these results being very close to ours.

During RV and MEE manoeuvres, IO, TA and RA showed a significant increase in thickness, which amounted to two- or threefold the resting value in some subjects, whereas EO did not. These data are in agreement with previous electromyographic studies which quantified abdominal muscle activation during similar conditions. Strohl et al. [6], using ultrasound, observed an increase in thickness in the order of $0.2-0.6 \mathrm{~cm}$ for TA and IO and less for EO, as well as electromyographic activation of EO, IO and TA during an expulsive manoeuvre. More recently, De Troyer et al. [5] studied activation of EO, TA and RA by means of needle electrodes during expiration from FRC to RV and during expulsive manoeuvres with the glottis closed. In both cases they found an increased amount of electromyographic activity of all the muscles. These data, demonstrating that during forced expiratory manoeuvres all the abdominal muscles are activated, suggest that the abdominal muscle thickening we observed is likely to be the result of active shortening. On the other hand, our data showing that EO did not change its thickness during RV and MEE manoeuvres differ somewhat from the results of De Troyer et al. [5]. A possible explanation of the discrepancy between electromyographic and ultrasonographic assessment of EO activity may lie in the fact that during RV and MEE manoeuvres, EO activation was not sufficient to induce a measurable change in muscle thickness.

During PEEs, TA thickness was closely related to the pressure developed, the thickness of the other abdominal muscles being inconsistently related to $P$ g. This observation deserves a comment. The major role played by TA contraction in increasing $P$ g could depend on the fact that, unlike other abdominal muscles, TA runs circumferentially around the abdominal cavity and thus is probably in a more favourable position and more effective in generating abdominal pressure [5].

Thickening might be expected to be greater during manoeuvres that produce the greatest shortening, i.e. RV. In contrast, during MEEs and at the highest pressure values during PEEs, increase in thickness was similar to that observed during RV. However, one must take into account the following: 1) it is well known that during isometric contraction, muscles shorten to a variable degree: in the human gastrocnemius in vivo during isometric contraction it has been reported that fibre length decreased from 50.8 to $32.9 \mathrm{~mm}$, i.e. a reduction of about $35 \%$, with no significant change in the distance between the aponeuroses [18]. It appears that during MEE and PEE manoeuvres, shortening and thickening of abdominal muscles is greater than would be expected if the contraction were truly isometric: in fact, due to thoracic gas compression, abdominal volume decreases and the contraction is not completely isometric; 2) muscles have three dimensions and as suggested for the diaphragm [16], the volume of a muscle $(V)$ is the product of its length (L), width (w), and thickness (t). If this holds also for abdominal muscles, assuming that $V$ is constant, L will vary inversely with w.t. We have no data on width or length of abdominal muscles, so we are not able to establish the exact relationship that links the three dimensions to each other. Similarly, the differences in shape, fibre direction and bone insertions of each abdominal muscle in vivo may further complicate the relationship between muscle thickness and the other dimensions during MEE and PEE; 3) our data are corroborated by and fully in agreement with a previous ultrasound study performed on the human diaphragm [10]. These authors showed similar, apparently conflicting, data, in that since during maximal inspiratory efforts (MIEs) at FRC against a closed airway and at TLC, they observed a similar degree of muscle thickening. In fact, the mean \pm SD diaphragm thickness at FRC was $1.7 \pm$ $0.9 \mathrm{~mm}$, whereas during MIEs and at TLC it rose to $4.4 \pm 1.4$ and $4.5 \pm 0.9 \mathrm{~mm}$, respectively, i.e. an approximately 2.6 fold increase. It is noteworthy that, in some subjects, the extent of thickening during MIEs was almost fourfold the resting value. Besides observing that the increase in thickness during MIEs was disproportionate to the likely thickening due to shortening, they adduced several hypotheses to explain this finding. For example, vascular changes during muscle contraction might play a relevant role, the vessels running between muscle fibres. It is evident that the same explanation may apply to our data. To what extent these factors play a role in muscle thickening is not known and further studies are required to establish the origin of the observed response.

We found that at TLC, IO and TA thickness decreased significantly, and EO and RA tended to be thinner, suggesting that the descent of the diaphragm induced a stretching of abdominal muscles at end-inspiration.

Finally, while IO appeared to have both a respiratory and a postural function, EO seemed to be mostly involved during trunk rotation and, compared to the other abdominal muscles, to have less mechanical effect during MEE manoeuvres.

In conclusion, ultrasound appears to be a useful technique which easily and noninvasively contributes to assessment of abdominal muscle function in humans. Our data indicate that the anterolateral wall of the abdomen does not operate as a unit during respiratory manoeuvres. Transversus abdominis seems to be the major contributor in generating gastric pressure during progressive voluntary expiratory efforts. These results suggest the possible value of studying the abdominal muscles by ultrasonography in various respiratory disorders.

\section{References}

1. Robertson CH Jr, Pagel MA, Johnson RL Jr. The distribution of blood flow, oxygen consumption, and work 
output among the respiratory muscles during unobstructed hyperventilation. J Clin Invest 1977; 59: 43-50.

2. Gilmartin JJ, Ninane V, De Troyer A. Abdominal muscle use during breathing in the anesthetized dog. Respir Physiol 1987; 70: 159-171.

3. De Troyer A, Gilmartin JJ, Ninane V. Abdominal muscle use during breathing in unanesthetized dogs. J Appl Physiol 1989; 66: 20-27.

4. Ninane V, Gilmartin JJ, De Troyer A. Changes in abdominal muscle length during breathing in supine dogs. Respir Physiol 1988; 73: 31-42.

5. De Troyer A, Estenne M, Ninane V, Van Gansbeke D, Gorini M. Transversus abdominis muscle function in humans. J Appl Physiol 1990; 68: 1010-1016.

6. Strohl KP, Mead J, Banzett RB, Loring SH, Kosch PC. Regional differences in abdominal muscle activity during various manoeuvers in humans. J Appl Physiol: Respirat Environ Exercise Physiol 1981; 51 (6): 1471-1476.

7. Wakai Y, Welsh MM, Leevers AM, Road JD. Expiratory muscle activity in the awake and sleeping human during lung inflation and hypercapnia. J Appl Physiol 1992; 72 (3): 881-887.

8. Ninane V, Rypens F, Yernault JC, De Troyer A. Abdominal muscle use during breathing in patients with chronic airflow obstruction. Am Rev Respir Dis 1992; 146: $16-21$.

9. Abe T, Noriyuki K, Naotaka Y, Tomoyuki T, Easton PA. Differential respiratory activity of four abdominal muscles in humans. J Appl Physiol 1996; 80: 1379-1389.

10. Ueki J, De Bruin PF, Pride NB. In vivo assessment of diaphragm contraction by ultrasound in normal subjects. Thorax 1995; 50: 1157-1161.

11. McCool FD, Conomos P, Benditt JO, Cohn D, Sherman $\mathrm{CB}$, Hoppin FG Jr. Maximal inspiratory pressures and dimensions of the diaphragm. Am J Respir Crit Care Med 1997; 155: 1329-1334.

12. Wait JL, Nahormek PA, Yost WT, Rochester DP. Diaphragmatic thickness-lung volume relationship in vivo. J Appl Physiol 1989; 67: 1560-1568.

13. Cohn D, Benditt JO, Eveloff S, McCool FD. Diaphragm thickness during inspiration. J Appl Physiol 1997; 83: 291-296.

14. Houston JG, Angus RM, Cowan MD, McMillan NC, Thomson NC. Ultrasound assessment of normal hemidiaphragmatic movement: relation to inspiratory volume. Thorax 1994; 49: 500-503.

15. Agostoni E, Rahn H. Abdominal and thoracic pressure at different lung volumes. J Appl Physiol 1960; 15: 1087-1092.

16. McCool FD, Hoppin FG Jr. Ultrasonography of the diaphragm. In: Roussos C, ed. The Thorax. New York, Marcel Dekker, 1995; pp. 1295-1311.

17. Partridge LD, Benton LA. Muscle, the motor. In: Brookhart JM, Mountcastle VB, eds. Handbook of Physiology, Section 1, The Nervous System, Vol. II, part 1. Bethesda, MD, American Physiological Society, 1981; pp. 43-106.

18. Narici MV, Binzoni T, Hiltbrand E, Fasel J, Terrier F, Cerretelli P. In vivo human gastrocnemius architecture with changing joint angle at rest and during graded isometric contraction. J Physiol 1996; 496: 287-297. 